\title{
JUURNAL.RU
}

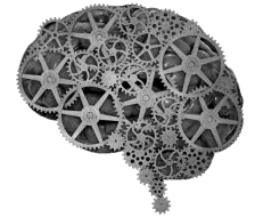

COMPANY GROUP "INTELLEKT"

\author{
Гушанский С.М., Потапов В.С. \\ Инженерная технологическая академия Южного федерального университета \\ Таганрог, Россия
}

doi: 10.18411/lj2016-7-1-05

idsp 000001: lj2016-16-1-05

\section{Выявление роли запутанности в построении и реализации квантовых алгоритмов}

Аннотация. В статье предполагается рассмотрение понятия квантовой запутанности чистых состояний, а также ее влияние на процесс и результат работы квантовых алгоритмов и квантовых систем. В работе анализируется роль запутанности в реализации квантовых алгоритмов и приводится, как частный случай, алгоритм Гровера. Описаны состояния полной запутанности в частном и общем виде. Отмечены основные преимущества использования квантовой запутанности в квантовых вычислениях и алгоритмах.Выполнены реализация и моделирование понятия квантовой запутанности с помощью программной модели реализации поискового алгоритма Гровера.

Ключевые слова: моделирование, запутанность, суперпозиция, запутанные состояния, квантовый алгоритм, кубит, модель квантового вычислителя.

\section{Введение}

В настоящее время огромное внимание уделяется разработкеметодов генерации запутанных квантовых состояний, а также манипуляции ими для практического использования запутанности. Однако экспериментальная реализация запутанных состояний и исследования их свойств - становятся все более и более сложными по мере увеличения числа частиц $\mathrm{N}$ в системе. 
Исследования запутанности квантово-механических состояний остается важным направлением изучения области квантовой теории информациипо причине значимости понятия запутанности в практической реализации алгоритмов и взаимосвязи этого понятия с разделами квантового компьютинга.

Одним из важнейших свойств запутанности, играющим важную роль в дальнейших рассуждениях, является инвариантность запутанности по отношению к локальным операциям над подсистемами квантовой системы.

1. Роль запутанности в реализации алгоритмаГровера

Пусть есть бинарная функция, состоящая из пбинарных аргументов, принимающая значение 1 только на одном из них, а на остальных (2n1) значенияхпринимает 0.

Задача заключается в нахождении этого значения входных аргументов, знаялишь функцию.

В классическом случае необходимо перебрать $2^{n} / 2$ вариантов входных значений, так какпри лучшем исходе удастся отыскатьнужный номер с первой попытки, а в худшем потребуется перебрать все 2пвариантов. Алгоритм Гровера позволяет выполнитьданный поискза $\frac{\pi}{4} * \sqrt{2^{n}}$ запросов.

Запутанность[1] - это особая форма корреляции квантовых частиц, не имеющая классических аналогов. В настоящее времяприлагаютсяогромные усилия, направленные на понимание этого физического явления, но теория запутанности еще окончательно не создана. Так, остаются нерешенными вопросы установления критериев запутанности квантового состояния; вопросы взаимосвязи различных мер запутанности; изучение общих свойств многокомпонентных квантовых систем. Состояния полной квантовой запутанности - состояния Белла[2]:

$$
\varphi^{ \pm}=\frac{1}{\sqrt{2}} *(|00\rangle \pm|11\rangle) \psi^{ \pm}=\frac{1}{\sqrt{2}} *(|01\rangle \pm|10\rangle)
$$


где $\varphi^{ \pm}-$ЭПР-состояние, а $\psi^{ \pm}-$состояние кота Шредингера. Эти четыре состояния Белла - лишь часть всех возможных состояний запутанности, их частный случай. Состояния запутанности в общем виде могут быть представлены следующими уравнениями:

$$
\varphi^{\alpha \pm}=\alpha^{*}|00\rangle \pm \beta^{*}|11\rangle \psi^{\alpha \pm}=\alpha^{*}|01\rangle \pm \beta^{*}|10\rangle
$$

Выведено уравнение определения универсальной меры запутанности, приложимой к чистым и смешанным состояниям[3]:

$$
S=\exp \left(i \frac{\beta}{2} \sigma_{1}^{\otimes N}\right)=\frac{1}{\sqrt{1+\beta^{2}}}\left(\sigma_{0} \otimes \sigma_{0}+i \beta \sigma_{1} \otimes \sigma_{1}\right),
$$

где $\beta$ - параметр определяющий степень запутанности кубит, устанавливается в интервале $[0 ; \pi / 2] . \mathrm{A}^{\sigma_{0}}{ }_{\text {и }} \sigma_{1}$ - матрицы Паули.Алгоритм Гровера[4] состоит из следующих этапов:

1. Переводим регистр в состоянии суперпозиции, то есть выравниваем вероятности всех $\mathrm{N}$ состояний. Выполнимость этого действия достигается за $\mathrm{O}(\log \mathrm{N})$ операций. В результате получим вектор, все компоненты которого равны.

2. Далее выполняем унитарные преобразования: $\mathrm{R}$ (преобразование поворота фазы) и D (преобразование диффузии) $\mathrm{O}(\log \mathrm{N})$ раз. После каждой итерации амплитуда нужного состояния изменится на величину ${ }^{2 *} M(\varphi)+\varphi_{i}$, где $M(\varphi)$ - среднее значение компонент вектора после преобразования R, a $\varphi_{i}$ - амплитуда желаемого состояния до R.

3. Измеряем состояние системы.

Квантовые алгоритмы благодаря использованию явления квантовой запутанности кубитов в процессе своей работы обладают значительным приростом скорости выполнения по сравнению с соответствующими классическими аналогами. 
Квантовая схема алгоритма зависит от размера входных данных, поскольку от этого напрямую зависит количество применений итерации Гровера.

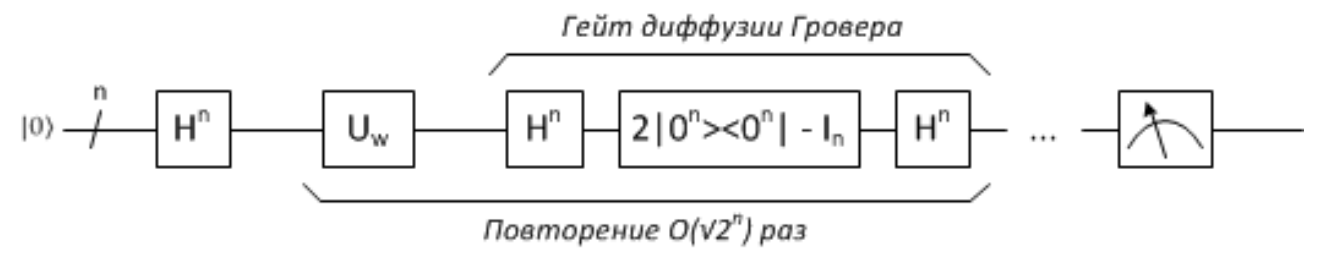

Рис. 1. Квантовая схема поискового алгоритма Гровера

Роль и преимущества квантовой запутанности:

1. Квантовые состояния двух или большего числа объектов оказываются взаимозависимыми, даже если эти объекты разнесены в пространстве за пределы любых известных взаимодействий. Данное явление получило название квантовой телепортации;

2. Способность информационных квантовых единиц- кубитов - принимать несколько значений одновременно и находиться в состоянии запутанности;

3. Возможность быстрого вычисления значенийквантовой системы с высокой точностью;

4. Квантовая криптография. Сложность и в большинстве случаев невозможность взлома криптосистемы. Передача открытых и закрытых ключей шифрования с помощью сцепленных частиц.

\section{2. Моделирование квантового алгоритма Гровера}

На рис.2представлена анимация квантового поискового алгоритма Гровера. Он находит х, для которых $\mathrm{P}(\mathrm{x})=1$, если предположить, что $\mathrm{n}$ равно 0 для всех остальных значений. В этом примере мы предполагаем, что х находится в диапазоне от 0 до 7 и, следовательно, нам нужно 3-разрядное пространство поиска. Пусть существует секретная функция F, которая выражена квантовым оракулом и удовлетворяет условию $\mathrm{P}(\mathrm{x})=1$ при $\mathrm{x}=2$.

На входе алгоритма имеем заранее определенное квантовое состояние. При нажатии на кнопку «Запустить» происходит соответственно запуск 
алгоритма, перевод регистра в состояние суперпозиции. В результате получаем вектор, все компоненты которого равны. Далее выполняется преобразование поворота фазы и преобразование диффузии для каждого состояния. Визуально это подтверждается изменением положения стрелок (векторов) внутри каждого круга (состояния). Другими словами, после каждой итерации амплитуда нужного состояния изменяется. Одна итерация заканчивается измерением состояния системы.

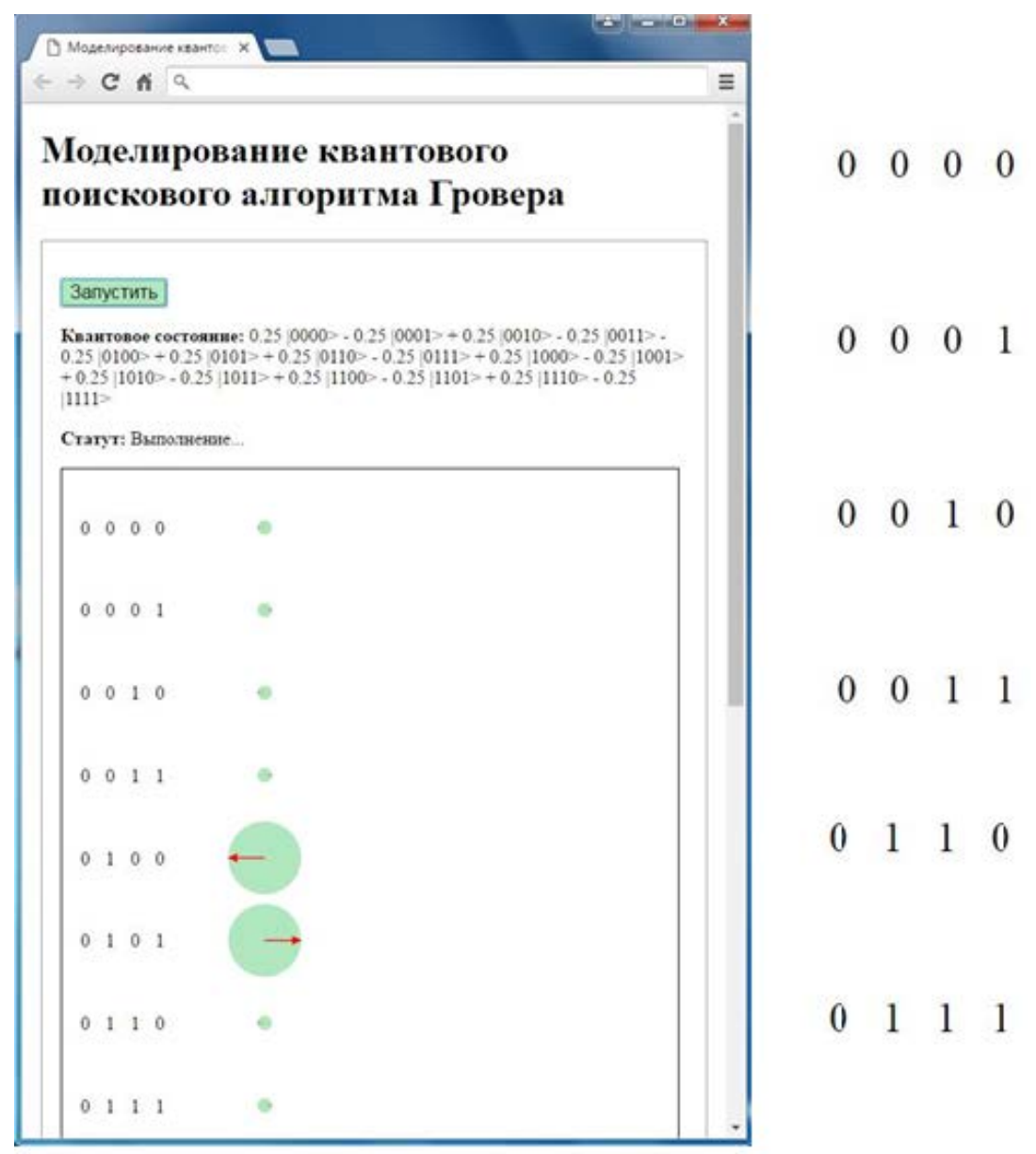

Рис. 2. Программная реализация квантового алгоритма Гровера

Геометрически, важной частью работы данного алгоритма является вращение текущего вектора (красные стрелки) состояния квантового вычислителя по направлению точно к целевому состоянию (движение по наикратчайшему пути обеспечивает оптимальность). Каждый шаг работы алгоритма дает вращение. Каждая итерация алгоритма визуализируется выдвижение вправо проверяемых на данный момент состояний, после завершения этого процесса, они возвращаются назад (Рис. 3). 

$\begin{array}{llll}0 & 0 & 1 & 0\end{array}$
$\begin{array}{llll}0 & 0 & 1 & 1\end{array}$
$\begin{array}{llll}0 & 1 & 0 & 0\end{array}$
$\begin{array}{llll}0 & 1 & 0 & 1\end{array}$

Рис. 3. Процесс проверки квантовых состояний

Алгоритм требует три кубита для поиска пространства и один дополнительный «рабочий» кубит, чтобы сделать возможным выполнение требуемых фазовых поворотов. Здесь три левых кубита составляют пространство поиска, а крайний правый является рабочим. Исходное состояние состоит из "0000", затем применяется оператор Адамара ко всем кубитам. Учитывая, что $\mathrm{P}(\mathrm{x})=1$ для двоичного значения "010", алгоритм должен значительно увеличить величину амплитуд "0100" и "0101". Тогда необходимо измерить три кубита слева и получить высокую вероятность нахождения требуемого ответа "010".

Модель [5] применяет алгоритм Гровера для заданного оракула заданное количество раз, а результатом её работы является гистограмма результатов алгоритма, то есть список пар вида: частота появления, результат. В результате был построен график (Рис. 4). По вертикали отображено количество запусков алгоритма, по горизонтали - вероятность появления того или иного результата. 


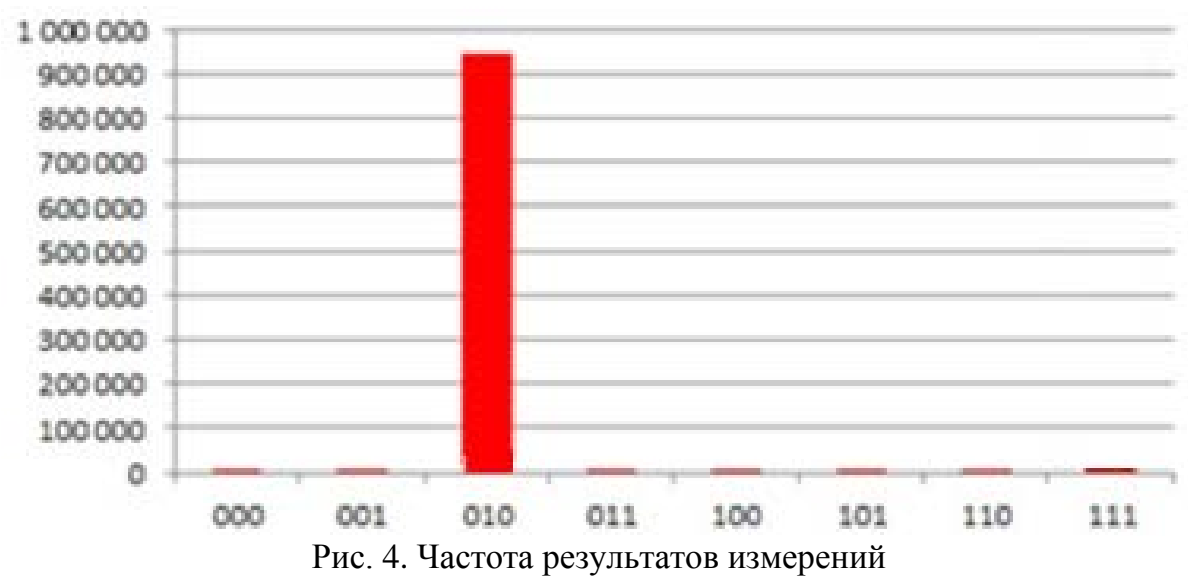

Необходимый результат получен примерно в 94.5\% запросов, а остальные результаты имеют частоту примерно в $0.78 \%$. Этого вполне достаточно для того, чтобы иметь возможность запустить алгоритм Гровера три раза и выбрать из этих трёх запусков результат, повторившийся, по крайней мере, дважды.

В соответствии со всем вышеописанным можно сделать логический и практический вывод. Они являются взаимосвязанными через модель (Рис. 2), в которой отражается и подтверждается, в силу своей реализации, большая часть из пунктов (1, 2 и 3$)$ о роли и преимуществ квантовой запутанности в терминах квантовых вычислений и алгоритмов. Модель предлагает практический выводдоказательство успешной работы данного квантового алгоритма и как следствие истинность свойств квантовой запутанности, описанных в пункте 1.

Заключение

В настоящее время повышенное внимание к квантовой запутанности обусловлено задачами практического характера, в которых квантовая запутанность является ресурсом реализаций квантовых алгоритмов (КА), разработки квантовых криптографических протоколов и др.

В данной работе было рассмотрено понятие квантовой запутанности чистых состояний, каким образом ее присутствие влияет на процесс и результат работы квантовых алгоритмов и квантовых систем, а также ее роль в реализации КА и, как частный пример, алгоритма Гровера. Описаны состояния полной запутанности в частном и общем виде. Отмечены основные преимущества использования квантовой запутанности в квантовых вычислениях и 
алгоритмах.Выполнены реализация и моделирование понятия квантовой запутанности с помощью программной модели реализации поискового алгоритма Гровера.

Работа выполнена при финансовой поддержске РФФИ, грант № НК 1501-01270. 


\section{Литература:}

1. Guzik V., Gushanskiy S., Polenov M., Potapov V. Architecture and Software Implementation of a Quantum Computer Model // 5th Computer Science Online Conference 2016, Czech Republic, 2016. - P. 59 - 68.

2. Не-белловские квантовые запутанные состояния // URL: http://samlib.ru/p/putenihin_p_w/non-bell.shtml (Дата обращения: 09.06.2016);

3. Гузик В.Ф., Гушанский С.М., Касаркин А.В. Использование квантовой запутанности для моделирования параметра согласованности в задачах теории игр // Известия ЮФУ. Технические науки. - Таганрог: Изд-во ТТИ ЮФУ, 2014. N 4 (153). - C. 22-28.

4. Алгоритм Гровера // URL: https://ru.wikipedia.org/wiki/ Алгоритм_Гровера (Дата обращения: 09.06.2016);

5. Потапов В.С., Гузик В.Ф., Гушанский С.М. Модель квантового вычислителя, архитектура и программная реализация // Информационные технологии, системный анализ и управление (ИТСАиУ-2014) / Сборник трудов XII Всероссийской научной конференции молодых ученых, аспирантов и студентов, г. Таганрог, 18-19 декабря 2014 г. - Ростов-наДону: Издательство Южного федерального университета, 2015 - Т.1. C.110-118. 